\title{
Physiological Genomics of Antidepressant Targets: Keeping the Periphery in Mind
}

\author{
Randy D. Blakely \\ Department of Pharmacology, Center for Molecular Neuroscience, Vanderbilt University School of Medicine, Nashville, \\ Tennessee 37232-6420
}

The plasma membrane transporters that clear extracellular serotonin (5-HT) and norepinephrine (NE), serotonin transporters (SERTs) and NE transporters (NETs), have received considerable attention over the past four decades because of their roles in amine neurotransmitter inactivation. In addition, they interact with many centrally active drugs, including multiple classes of antidepressants such as the serotonin-selective reuptake inhibitors, typified by fluoxetine (Prozac), and the more recently developed norepinephrine-selective transporter antagonists, such as reboxetine. The therapeutic utility of these agents supports biogenic amine theories of affective disorders and raises the question as to whether SERT and NET exhibit a functional genetic variation that could influence risk for behavioral disorders. Although evidence exists that a promoter polymorphism in SERT may influence behavioral states, this contention is not without complexity and its mechanism of action remains poorly understood. The identification of coding vari- ants of NETs and SERTs would offer important opportunities to connect genotype to phenotype. However, given the limited frequency of transporter coding variations evident to date in general population surveys or in psychiatric genetic studies, the identification of informative functional variants of transporters will likely require refined phenotypes. In this regard, NET and SERT play critical roles in cardiovascular and gastrointestinal physiology, respectively. This perspective reviews recent human and mouse studies that suggest how peripheral autonomic phenotypes, linked to genetic disruption of NET and SERT function, can aid in the phenotypic segregation needed for advanced theories of biogenic amine dysfunction and pharmacogenetics.

Key words: norepinephrine; serotonin; transport; antidepressant; genetics; orthostatic intolerance; irritable bowel syndrome; serotonin-selective reuptake inhibitor; polymorphism, single-nucleotide polymorphism
Ashcroft's wonderful compilation of ion channel defects that underlie clinical syndromes (Ashcroft, 2000), the so-called "channelopathies," frustrates me to no end. Of course, ion channels support all fundamental aspects of membrane biology and without ion channels, the nervous system would grow as quiet as a church mouse. But chemical signaling in the brain and periphery also uses another class of channel-like proteins, the neurotransmitter transporters (Barker and Blakely, 1995; Masson et al., 1999), that do the heavy lifting in neurotransmitter inactivation. As one devoted to studying the structure and regulation of these transporters, particularly the antidepressant-sensitive norepinephrine (NE) transporters (NETs) and serotonin (5-HT) transporters (SERTs), I am puzzled that only a single, validated coding polymorphism has been identified that supports a clinical syndrome (Shannon et al., 2000). Perhaps to date we have only looked for genetic transporter dysfunctions in the light cast by past research in psychopharmacology, rather than by following

I am supported by National Institute of Mental Health Grant MH58921, by National Institute on Drug Abuse Grant DA07390, by National Institute of Diabetes and Digestive and Kidney Diseases Grant DK58212, and by the National Parkinson's Foundation. I acknowledge the vital collaborations of John Shannon, Nancy Flattem, David Robertson, Richard Shelton, Howard Mertz, and Michael Gershon in developing the research area on which this perspective is based as well as the ongoing efforts of Maureen Hahn, Alexandra Belous, and Michelle Mazei to define the clinical impact of transporter genetic variation.

Correspondence should be addressed to Dr. Randy D. Blakely, Center for Molecular Neuroscience, 417 Preston Research Building, Vanderbilt School of Medicine, Nashville, TN 37232-6420. E-mail: randy.blakely@mcmail. vanderbilt.edu.

Copyright (C) 2001 Society for Neuroscience $0270-6474 / 01 / 218319-05 \$ 15.00 / 0$ leads taken from more systemic considerations of NE and 5-HT (and their transporters) actions throughout the body. In my view, those in the hunt for transporter contributions to disease should perhaps think "outside the brain" to develop richer, more biologically consistent phenotypes linked to amine distribution and function in the periphery.

\section{Genetic support for NET dysfunction: "you gotta have heart"}

The human NET was the first of the biogenic amine transporters to be cloned and characterized (Pacholczyk et al., 1991), apropos given that NETs were the first neurotransmitter transporters to be understood in terms of a contribution to synaptic signaling and drug response. After decades of physiologic and pharmacologic studies, we entered the 1990s knowing that at some synapses NETs can recover as much as $90 \%$ of released NE and that NETs are high-affinity targets for cocaine and secondary amine tricyclic antidepressants, typified by desipramine. With the cloning studies, we learned that NET proteins possess 12 transmembrane domains (TMDs) and that a single cDNA could generate fully functional catecholamine transporters once transfected into cell hosts, complete with cocaine, amphetamine, and tricyclic antidepressant sensitivity. Subsequently, a single NET gene (SLC6A2) was identified and mapped to chromosome 16q12.2 (Brüss et al., 1993; Gelernter et al., 1993b). For those hoping to identify genetic disorders supported by NET loss- or gain-of-function mutations, a single gene is ideal because it limits opportunities for compensation by closely related subtypes. The related dopamine 


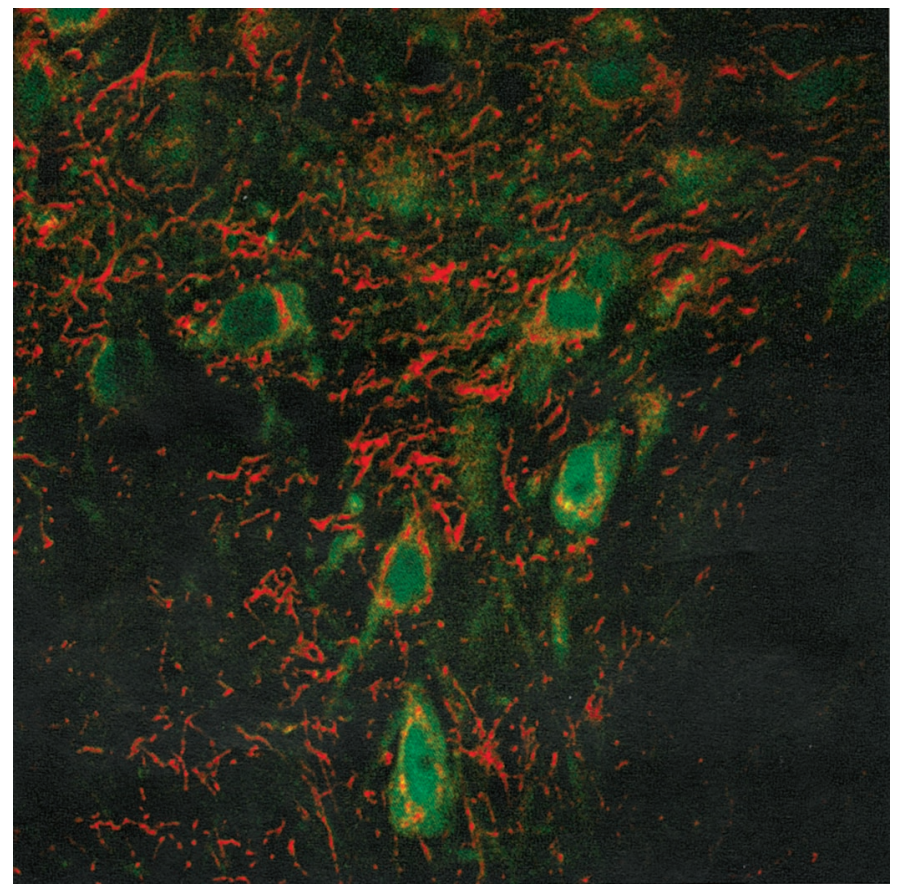

Fig 1. Serotonin and SERT localization in the dorsal raphe. A fluorescence micrograph of serotonin (green) and SERT (red) immunoreactivity is shown (S. Schroeter and R. D. Blakely).

transporter (DAT) can transport NE (Buck and Amara, 1994); however, DATs are not expressed in central or peripheral noradrenergic neurons (Lorang et al., 1993).

After the isolation and mapping of the human NET gene, several noncoding and coding polymorphisms were identified, but none showed association with bipolar disorder or major depression (Gelernter et al., 1993a; Hadley et al., 1995) or with schizophrenia or Tourette's syndrome (Stöber et al., 1996, 1999). Five single-nucleotide polymorphisms (SNPs), known as "nonsynonymous SNPs," were found to change the coding sequence of NETs, but these variants fail to markedly influence NET function in vitro. Possibly, these variants could perturb NET function or regulation but might require more complex interrogation, such as response to drugs that alter noradrenergic signaling. In this regard, NET knock-out mice are not overtly distinct from their wild-type littermates but can be distinguished behaviorally when stressed or challenged pharmacologically (hypersensitivity to psychostimulants, opiates) (Bohn et al., 2000; Xu et al., 2000). Possibly, the identified variants could support physiological alterations, but the critical physiological system has yet to be queried.

The fact that a single gene encodes NETs in the CNS and periphery means that any coding polymorphisms have the potential to influence NE clearance in both the central and autonomic nervous systems. Because the complexity and plasticity of the CNS might mute many of the influences of NET genetic variation altogether, we have turned our attention to the noradrenergic control of heart rate, where NET dysfunction might be expected to elicit a quantifiable and clinically relevant phenotype. NETs have long been implicated in the tight control of synaptic NE in the heart (Esler et al., 1990; Eisenhofer et al., 1991). Moreover, Robertson (1999) brought to our attention patients presenting with orthostatic intolerance (OI), an autonomic disorder of perhaps half a million Americans, mostly women. OI (Jacob and
Biaggioni, 1999; Robertson, 1999) is characterized by sustained tachycardia on standing ( $>30$ beats per minute) without a fall in blood pressure (orthostatic hypotension) and is often accompanied by chronic fatigue, headache, dizziness, and syncope (fainting). Studies by Robertson's group demonstrated that OI in a set of twins resulted in signs of NET dysfunction [e.g., elevated plasma NE spillover, decreased NE disappearance (clearance), and insensitivity to the indirect agonist tyramine]. We sequenced the exons and splice junctions of the NET gene in one of the probands and found a single coding mutation: a $\mathrm{G}$ to $\mathrm{C}$ transversion that results in a proline for alanine substitution in a highly conserved region of NET TMD 9 (Shannon et al., 2000). Studies of the mutant NET in vitro reveal an essentially complete loss of function as well as a failure of NET protein to mature and reach the surface in normal levels (M. Hahn and R. D. Blakely, unpublished observations). Analysis of the relatives of the initial probands revealed that tilt-induced tachycardia and plasma catecholamine abnormalities track the presence of the mutant allele (Shannon et al., 2000). We have found recently that NET A457P can negatively impact the surface expression of wild-type NET proteins in cotransfection studies, perhaps explaining how the heterozygosity of our probands results in such a profound phenotype (M. Hahn and R. D. Blakely, unpublished observations). We suspect that other subjects with OI-like phenotypes may harbor additional nonsynonymous NET mutations or have alterations in critical regulatory regions.

OI, like depression or anxiety, is also likely to be a heterogeneous disorder with multiple genetic and environmental determinants. Indeed it is likely that OI in general is not attributable to a NET defect. Rather, OI-like phenotypes may arise from a number of other control points in NE synthesis, packaging, release, and response. What is clear is that NET deficiency can present with OI-like symptoms. Thus, as studies progress with NET as a candidate gene, culling out subjects on the basis of cardiovascular symptoms, particularly those evident in our OI study, would appear valuable in identifying or associating NET polymorphisms. In fact, Halushka et al. (1999) more than doubled the known coding polymorphisms in NET using subjects collected on the basis of blood-pressure abnormalities. One of these variants shows a trafficking abnormality and loss of function like A457P (M. Hahn and R. D. Blakely, unpublished observations), whereas another displays a gain of function that could hold important clues as to how transport activity is normally constrained. In this regard, regulatory stimuli have been shown to downregulate transporter proteins in vitro via altered surface trafficking, and many sites in vivo have significant cytoplasmic pools of NET protein (Blakely and Bauman, 2000; Schroeter et al., 2000). Perhaps novel coding variants display altered regulation, revealing important facets of regulatory biology that may be influenced in disease states. Together these studies remind us that although a gene such as NET may be expressed in multiple tissues, one locale may be more visibly compromised than another. Making strategic choices based on a more global physiologic assessment can reveal enriched populations to mine for candidate gene variants. Studies of families with functional variants can then in turn lead to a richer classification of clinical phenotypes.

\section{Serotonin transporters: a gut reaction to the search for genetic variants}

SERTs reside in the same gene family as NETs and are also high-affinity targets of antidepressant medications (Ramamoorthy 
et al., 1993; Tatsumi et al., 1997). Altered SERT expression or function has long been suggested to contribute to anxiety and affective disorders (Owens and Nemeroff, 1994), and serotoninselective reuptake inhibitors have found extensive use in the treatment of depression, anxiety disorders, obsessive-compulsive disorder (OCD), and certain aspects of substance abuse. As with NET, a single gene (SLC6A4, 5-HTT), located in this case at 17q11.2 (Ramamoorthy et al., 1993), encodes SERTs in the CNS (Fig. 1) and periphery. Genetic variation in the SERT promoter (5HTTLPR) has received substantial attention as a potential risk factor for anxiety, alcoholism, and mood disorders (VeenstraVanderWeele et al., 2000) ever since Lesch et al. (1996) revealed its polymorphic character and documented both a correlation with functional expression of SERT mRNAs in lymphocytes and an association with neuroticism and anxiety traits. The model proposed in these initial studies was that the short variant (fewer repeats) of the 5HTTLPR yields less robust mRNA transcription than the long variant and presumably diminished 5-HT clearance in vivo. Recently, Mundo et al. (2001) have noted that the 5HTTLPR influences risk for mania in bipolar subjects treated with antidepressants, suggesting that drug-targeted phenotypes may be an exciting area for future analyses. The degree to which the 5HTTLPR influences SERT expression in vivo is controversial, with both supportive (Little et al., 1998; Heinz et al., 2000) and contradictory (Willeit et al., 2001) evidence. The identification of genetic variants that actually change the structure of SERT proteins and lead to demonstrable functional variation in 5-HT transport activity would be exceedingly useful in understanding how SERT dysfunction supports disease states.

To date studies searching for coding variants of SERT proteins have focused either on normal subjects or on psychiatric diagnoses. In initial studies, no coding variants were found in SERTs with respect to OCD (Altemus et al., 1996), major depression, or bipolar disorder (Lesch et al., 1995). In these studies, which focused exclusively on psychiatric abnormalities, only a single synonymous SNP in one bipolar patient was observed. In a recent SNP survey study of candidate genes for cardiovascular (unfortunately no SNPs in NET were explored), hormonal, or neuropsychiatric disorders, Cargill et al. (1999) identified four new SNPs for SERT. Of the coding variants described, one results in an alanine for glycine substitution at amino acid 77 in the NH2 terminus of the transporter, whereas the second is a nonconservative substitution of an aspartate for a lysine residue at amino acid 605 in the SERT COOH terminus. Neither of these variants has yet to be explored for functional impact or association with a specific disorder. Their location in cytoplasmic domains suggests that they could influence transporter regulation. Recently, Glatt et al. (2001) sought evidence for SERT variation in a large (450 subjects, 900 chromosomes) panel of clinically uncharacterized subjects. As in the other studies noted, coding variants were exceedingly rare (all $<1 \%$ ), although a new set of SERT coding variants was identified. The SERT gene, like that of NET, is composed of more than a dozen exons comprising $\sim 2 \mathrm{~kb}$ of sequence, but stretched out by introns across $>40 \mathrm{~kb}$ of genomic sequence. Possibly this distribution of exons results in fewer exonic alterations than seen in intronic regions. Eight of the nine coding variants in the study by Glatt et al. (2001) were found to be associated only with a single chromosome, whereas the ninth was found only on four chromosomes.

From these studies, one might conclude that coding variants in SERTs are rare and perhaps of limited value for additional investigation. Although rare, these variants could harbor important information as to how structural variation limits function, drug interactions, or SERT regulation, lessons that could influence subsequent studies in more defined populations. Moreover, by analogy to our NET studies in OI, even a single family with traits defined by functionally validated transporter alleles can provide fundamental insights into transporter structure and processing and also sharpen phenotypic classifications for the benefit of patients and therapies. Certainly a realistic interpretation of the low frequency of coding alleles is that meaningful SERT coding variants will only be rarely detected without a priori phenotypic enrichment. I contend that for SERT, like NET, we can obtain the needed subject enrichment using inclusion criteria that derive from a more peripheral view of transporter function.

5-HT plays a number of important roles outside the CNS (Fozzard, 1989). 5-HT is secreted in copious amounts from gut enterochromaffin cells and serves as a critical messenger for gastrointestinal (GI) fluid secretion and gut motility (Ormsbee and Fondacaro, 1985; Gershon, 1999). Disruption of GI 5-HT function may underlie components of irritable bowel syndrome (IBS) (Sanger, 1996; Crowell, 2001). Indeed, until its recent removal from the market by the Unites States Food and Drug Administration, alosetron, a 5-HT-3 receptor antagonist, was on the front line in the treatment of IBS, suggesting that the disorder may arise from or result in hyperserotonergic signaling in the gut. To control 5-HT actions in the gut and limit 5-HT receptor desensitization, both neurons and crypt epithelial cells synthesize SERT proteins (Wade et al., 1996; Chen et al., 1998). Moreover, SERT knock-out mice are not only anxious when examined in standardized behavioral tests (A. Holmes et. al., personal communication) but also exhibit altered GI function reminiscent of IBS (Chen et al., 2001). It seems reasonable therefore to suggest that studies of psychiatric disorders hypothesizing SERT alterations may benefit from a careful consideration of GI comorbidity. The thesis is not that IBS is equivalent to SERT dysfunction but rather that SERT dysfunction should present just as likely with a GI phenotype as a behavioral disorder. In line with this notion, anxiety disorders are commonly observed to be comorbid with IBS (Walker et al., 1992; Addolorato et al., 1998; Woodman et al., 1998) and gastrointestinal side effects can accompany antidepressant treatment. SERTs are also found in other peripheral sites including platelets (Lesch et al., 1993), the pulmonary endothelium (Lee and Fanburg, 1986), and the placenta (Balkovetz et al., 1989). Indeed, human SERTs were initially cloned from the placenta (Ramamoorthy et al., 1993). A greater understanding of the role of placental 5-HT may add an additional peripheral phenotype, in addition to functional bowel disorders, for the evaluation of clinically relevant SERT variation.

In summary, the hunt for functional coding variants in NET has benefited from orienting genetic studies toward cardiovascular phenotypes. Studies exploring SERT contributions to psychiatric disorders would likely benefit from considering the important roles of 5-HT in the periphery, particularly in sustaining GI function. Even for DATs, where overt hyperactivity of knock-out mice (Giros et al., 1996) suggests that tracking behavioral perturbations alone might be sufficient to reveal novel transporter alleles in human hyperactivity syndromes, increasing evidence points to a role for this transporter in the periphery (Mezey et 
al., 1996; Chen et al., 2001). Given the highly distributed exons of amine transporter genes, we should not be so surprised by the rarity of coding variants in the general population. However, it would not be wise to dismiss the opportunity to identify important and revealing functional variants of transporter proteins until the most biologically consistent phenotypes have been surveyed. I know it in my heart and feel it in my gut.

\section{REFERENCES}

Addolorato G, Marsigli L, Capristo E, Caputo F, Dall'Aglio C, Baudanza P (1998) Anxiety and depression: a common feature of health careseeking patients with irritable bowel syndrome and food allergy. Hepatogastroenterology 45:1559-1564.

Altemus M, Murphy DL, Greenberg B, Lesch KP (1996) Intact coding region of the serotonin transporter gene in obsessive-compulsive disorder. Am J Med Genet 67:409-411.

Ashcroft FM (2000) Ion channels and disease. San Diego: Academic.

Balkovetz DF, Tiruppathi C, Leibach FH, Mahesh VB, Ganapathy V (1989) Evidence for an imipramine-sensitive serotonin transporter in human placental brush-border membranes. J Biol Chem 264:2195-2198.

Barker EL, Blakely RD (1995) Norepinephrine and serotonin transporters: molecular targets of antidepressant drugs. In: Psychopharmacology: the fourth generation of progress (Bloom FE, Kupfer DJ, eds), pp 321-333. New York: Raven.

Blakely RD, Bauman AL (2000) Biogenic amine transporters: regulation in flux. Curr Opin Neurobiol 10:328-336.

Bohn LM, Xu F, Gainetdinov RR, Caron MG (2000) Potentiated opioid analgesia in norepinephrine transporter knock-out mice. J Neurosci 20:9040-9045.

Brüss M, Kunz J, Lingen B, Bönisch H (1993) Chromosomal mapping of the human gene for the tricyclic antidepressant-sensitive noradrenaline transporter. Hum Genet 91:278-280.

Buck KJ, Amara SG (1994) Chimeric dopamine-norepinephrine transporters delineate structural domains influencing selectivity for catecholamines and 1-methyl-4-phenylpyridinium. Proc Natl Acad Sci USA 91:12584-12588.

Cargill M, Altshuler D, Ireland J, Sklar P, Ardlie K, Patil N, Lane CR, Lim EP, Kalayanaraman N, Nemesh J, Ziaugra L, Friedland L, Rolfe A, Warrington J, Lipshutz R, Daley GQ, Lander ES (1999) Characterization of single-nucleotide polymorphisms in coding regions of human genes. Nat Genet 22:231-238.

Chen JJ, Li Z, Pan H, Murphy DL, Tamir H, Koepsell H, Gershon MD (2001) Maintenance of serotonin in the intestinal mucosa and ganglia of mice that lack the high-affinity serotonin transporter: abnormal intestinal motility and the expression of cation transporters. J Neurosci 21:6348-6361.

Chen JX, Pan H, Rothman TP, Wade PR, Gershon MD (1998) Guinea pig 5-HT transporter: cloning, expression, distribution, and function in intestinal sensory reception. Am J Physiol 275:G433-G448.

Crowell MD (2001) The role of serotonin in the pathophysiology of irritable bowel syndrome. Am J Manag Care 7:S252-S260.

Eisenhofer G, Esler MD, Meredith IT, Ferrier C, Lambert G, Jennings G (1991) Neuronal re-uptake of noradrenaline by sympathetic nerves in humans. Clin Sci (Colch) 80:257-263.

Esler M, Jennings G, Lambert G, Meredith I, Horne M, Eisenhofer G (1990) Overflow of catecholamine neurotransmitters to the circulation: source, fate, and functions. Physiol Rev 70:963-985.

Fozzard JE (1989) Peripheral actions of 5-hydroxytryptamine. New York: Oxford UP.

Gelernter J, Kruger S, Kidd KK, Amara S (1993a) Taql RFLP at norepinephrine transporter protein (NET) locus. Hum Mol Genet 2:820.

Gelernter J, Kruger S, Pakstis AJ, Pacholczyk T, Sparkes RS, Kidd KK, Amara SG (1993b) Assignment of the norepinephrine transporter protein (NET1) locus to chromosome 16. Genomics 18:690-692.

Gershon MD (1999) Review article: roles played by 5-hydroxytryptamine in the physiology of the bowel. Aliment Pharmacol Ther 13 [Suppl 2]:15-30.

Giros B, Jaber M, Jones SR, Wightman RM, Caron MG (1996) Hyperlocomotion and indifference to cocaine and amphetamine in mice lacking the dopamine transporter. Nature 379:606-612.

Glatt CE, DeYoung JA, Delgado S, Service SK, Giacomini KM, Edwards RH, Risch N, Freimer NB (2001) Screening a large reference sample to identify very low frequency sequence variants: comparisons between two genes. Nat Genet 27:435-438.

Hadley D, Hoff M, Holik J, Reimherr F, Wender P, Coon H, Byerley W
(1995) Manic-depression and the norepinephrine transporter gene. Hum Hered 45:165-168.

Halushka MK, Fan JB, Bentley K, Hsie L, Shen N, Weder A, Cooper R, Lipshutz R, Chakravarti A (1999) Patterns of single-nucleotide polymorphisms in candidate genes for blood-pressure homeostasis. Nat Genet 22:239-247.

Heinz A, Jones DW, Mazzanti C, Goldman D, Ragan P, Hommer D, Linnoila M, Weinberger DR (2000) A relationship between serotonin transporter genotype and in vivo protein expression and alcohol neurotoxicity. Biol Psychiatry 47:643-649.

Jacob G, Biaggioni I (1999) Idiopathic orthostatic intolerance and postural tachycardia syndromes. Am J Med Sci 317:88-101.

Lee S, Fanburg BL (1986) Serotonin uptake by bovine pulmonary artery endothelial cells in culture. I. Characterization. Am J Physiol 250:C761-C765.

Lesch KP, Wolozin BL, Murphy DL, Riederer P (1993) Primary structure of the human platelet serotonin uptake site: identity with the brain serotonin transporter. J Neurochem 60:2319-2322.

Lesch KP, Gross J, Franzek E, Wolozin BL, Riederer P, Murphy DL (1995) Primary structure of the serotonin transporter in unipolar depression and bipolar disorder. Biol Psychiatry 37:215-223.

Lesch KP, Bengel D, Heils A, Sabol SZ, Greenberg BD, Petri S, Benjamin J, Müller CR, Hamer DH, Murphy DL (1996) Association of anxiety-related traits with a polymorphism in the serotonin transporter gene regulatory region. Science 274:1527-1531.

Little KY, McLaughlin DP, Zhang L, Livermore CS, Dalack GW, McFinton PR, DelProposto ZS, Hill E, Cassin BJ, Watson SJ, Cook EH (1998) Cocaine, ethanol, and genotype effects on human midbrain serotonin transporter binding sites and mRNA levels. Am J Psychiatry 155:207-213.

Lorang D, Amara SG, Simerly RB (1993) Cell-type specific expression of catecholamine transporters in the rat brain. J Neurosci 14 4903-4914.

Masson J, Sagn C, Hamon M, Mestikawy SE (1999) Neurotransmitter transporters in the central nervous system. Pharmacol Rev 51:439-464.

Mezey E, Eisenhofer G, Harta G, Hansson S, Gould L, Hunyady B, Hoffman BJ (1996) A novel nonneuronal catecholaminergic system exocrine pancreas synthesizes and releases dopamine. Proc Natl Acad Sci USA 93:10377-10382.

Mundo E, Walker M, Cate T, Macciardi F, Kennedy JL (2001) The role of serotonin transporter protein gene in antidepressant-induced mania in bipolar disorder: preliminary findings. Arch Gen Psychiatry 58:539-544.

Ormsbee III HS, Fondacaro JD (1985) Action of serotonin on the gastrointestinal tract. Proc Soc Exp Biol Med 178:333-338.

Owens MJ, Nemeroff CB (1994) Role of serotonin in the pathophysiology of depression: focus on the serotonin transporter. Clin Chem 40:288-295.

Pacholczyk T, Blakely RD, Amara SG (1991) Expression cloning of a cocaine- and antidepressant-sensitive human noradrenaline transporter. Nature 350:350-354.

Ramamoorthy S, Bauman AL, Moore KR, Han H, Yang-Feng T, Chang AS, Ganapathy V, Blakely RD (1993) Antidepressant- and cocaine-sensitive human serotonin transporter: molecular cloning, expression, and chromosomal localization. Proc Natl Acad Sci USA 90:2542-2546.

Robertson D (1999) The epidemic of orthostatic tachycardia and orthostatic intolerance. Am J Med Sci 317:75-77.

Sanger GJ (1996) 5-Hydroxytryptamine and functional bowel disorders. Neurogastroenterol Motil 8:319-331.

Schroeter S, Apparsundaram S, Wiley RG, Miner LH, Sesack SR, Blakely RD (2000) Immunolocalization of the cocaine- and antidepressant-sensitive 1-norepinephrine transporter. J Comp Neurol 420:211-232.

Shannon JR, Flattem NL, Jordan J, Jacob G, Black B, Biaggioni I, Blakely $\mathrm{RD}$, Robertson D (2000) Orthostatic intolerance and tachycardia associated with norepinephrine-transporter deficiency. N Engl J Med 342:541-549.

Stöber G, Nothen MM, Pörzgen P, Brüss M, Bönisch H, Knapp M, Beckman H, Propping P (1996) Systematic search for variation in the human norepinephrine transporter gene: identification of five naturally occurring missense mutations and study of association with major psychiatric disorders. Am J Med Genet 67:523-532.

Stöber G, Hebebrand J, Cichon S, Brüss M, Bönisch H, Lehmkuhl G, Poustka F, Schmidt M, Remschmidt H, Propping P, Nothen MM (1999) Tourette syndrome and the norepinephrine transporter gene: results of systematic mutation screening. Am J Med Genet 88:158-163.

Tatsumi M, Groshan K, Blakely RD, Richelson E (1997) Pharmacological profile of antidepressants and related compounds at human monoamine transporters. Eur J Pharmacol 340:249-258. 
Veenstra-VanderWeele J, Anderson GM, Cook Jr EH (2000) Pharmacogenetics and the serotonin system: initial studies and future directions. Eur J Pharmacol 410:165-181.

Wade PR, Chen J, Jaffe B, Kassem IS, Blakely RD, Gershon MD (1996) Localization and function of a 5-HT transporter in crypt epithelia of the gastrointestinal tract. J Neurosci 16:2352-2364.

Walker EA, Katon WJ, Jemelka RP, Roy-Bryne PP (1992) Comorbidity of gastrointestinal complaints, depression, and anxiety in the Epidemiologic Catchment Area (ECA) Study. Am J Med 92:26S-30S.

Willeit M, Stastny J, Pirker W, Praschak-Rieder N, Neumeister A, Asen- baum S, Tauscher J, Fuchs K, Sieghart W, Hornik K, Aschauer HN, Brucke T, Kasper S (2001) No evidence for in vivo regulation of midbrain serotonin transporter availability by serotonin transporter promoter gene polymorphism. Biol Psychiatry 50:8-12.

Woodman CL, Breen K, Noyes Jr R, Moss C, Fagerholm R, Yagla SJ, Summers R (1998) The relationship between irritable bowel syndrome and psychiatric illness. A family study. Psychosomatics 39:45-54.

Xu F, Gainetdinov RR, Wetsel WC, Jones SR, Bohn LM, Miller GW, Wang YM, Caron MG (2000) Mice lacking the norepinephrine transporter are supersensitive to psychostimulants. Nat Neurosci 3:465-471. 